\title{
CORRELATION BETWEEN THE ELECTRONIC STRUCTURE OF QUINAZOLINE DERIVATIVES AND THE ACTIVITY OF THE NTR1 RECEPTOR
}

\author{
CARLOS SOLOAGA ARDILES*, CRISTIAN CASTRO RODRIGUEZ AND JULIO SURCO LUQUE
}

\author{
Departamento de Química, Universidad de Tarapacá, Av. General Velásquez 1775, P.O. Box 7-D Arica, Chile.
}

\begin{abstract}
The relationship between ectopic neurotensin expression (NTS) and tumor carcinoma invasion has produced studies that point to allosteric modulation of the regular NTR1 receptor. The use of quinazoline-derived drugs has shown excellent results in the regulation of the biological process mentioned. This study aims to establish the relationship between the electronic structure of quinazoline derivatives and the biological activity (expressed as EC50) that process in the NTR1 receptor, to propose a 2D pharmacophore. For this purpose, the Klopman-Peradejordi-Gómez (KPG) methodology was used. Calculations are included within the functional density theory (DFT) using the B3LYP / 631G theory level $(\mathrm{d}, \mathrm{p})$. The results concerning the biological activity are mainly driven by the interactions at the orbitalorbital level and by charges. These results can be used to propose new quinazoline derivatives with a better response in allosteric modulation of the NTR1 receptor.
\end{abstract}

Keywords: NTR1 receptor, quinazoline derivatives, KPG method (QSAR).

\section{INTRODUCTION}

Neurotensin (NT), a tridecapeptide of the brain-intestine, has a dual role as a neurotransmitter or neuromodulator in the central nervous system (CNS) [1]. The actions of NT and related signal transduction depend on the recognition of the peptide in the plasma membrane of the cells by three specific receptors, called NTR1, NTR2, and NTR3 [2]. For non-neuronal systems, the main roles of neurotensin in the gastrointestinal and cardiovascular system have been demonstrated [3]. Neurotensin is located in the intestinal mucosa (endocrine $\mathrm{N}$ cells), where it is believed to be involved in intestinal contraction [4,5]. The effects of NTS are mediated by three subtypes of neurotensin receptors (NTR), those already mentioned; NTR1, NTR2, and SORT1 [6]. Among these receptors, NTR1 mediates most of the biological functions of NTS. This is a receptor coupled to the $\mathrm{G}$ protein that is widely expressed throughout the central nervous system, where it acts as a neuromodulator $[7,8]$. Some studies show a significant relationship between ectopic expression of NTS and tumor invasion in hepatocellular carcinoma (HCC). In primary CHC tissues, the co-expression of NTS and neurotensin receptor 1 is a poor prognostic factor related to aggressive biological behaviors and poor clinical prognosis $[8,9]$. Of the three subtypes of neurotensin receptors (NTR), the NTR1 receptor is the most widely studied. This receptor mediates most of the known neurotensin effects, which makes it an interesting therapeutic objective. Despite the therapeutic promise of NTR1, the development of ligands for this receptor has been hindered [10]. Results have been reported, in which the authors propose a series of quinazoline-derived compounds, which showed a good response in allosteric modulation in the NTR1 receptor [4].

The present work aims to relate the electronic structure of quinazoline derivatives and biological activity (expressed as EC50) in the NTR1 receptor, to improve ligands that favor allosteric modulation in this receptor. The KPG (Klopman-Peradejordi-Gómez) method will be used, which employs local reactivity indices, based on quantum chemistry calculations and multiple linear regression statistics (LMRA) [11]. This method has been widely used when seeking to propose a $2 \mathrm{D}$ pharmacophore, which specifies not only the atoms involved but also the nature of the type of binding between the ligand and the receptor [12-14].

\section{METHODS, MODELS, AND CALCULATIONS}

The KPG method has been previously reported in several articles. In order not to extend in its explanation, the reader is left with some bibliographical references for further study [14-17]. This method is based on the following general equation: $\log (B A)=a+b M_{D_{i}}+c \log \left[\sigma_{D_{i}} /(A B C)^{1 / 2}\right]+\sum_{j}\left[e_{j} Q_{j}+f_{j} S_{j}^{E}+s_{j} S_{j}^{N}\right]+\sum_{j} \sum_{m}\left[h_{j}(m) F_{j}(m)+x_{j}(m) S_{j}^{E}(m)\right] \quad(1)$
$++\sum_{j} \sum_{m}\left[r_{j}\left(m^{\prime}\right) F_{j}\left(m^{\prime}\right)+t_{j}\left(m^{\prime}\right) S_{j}^{N}\left(m^{\prime}\right)\right]+\sum_{j}\left[g_{j} \mu_{j}+k_{j} \eta_{j}+o_{j} \omega_{j}+z_{j} S_{j}+w_{j} Q_{j}^{\max }\right]$

where $a, b, c, e_{j}, f_{j}, s_{j}, h_{j}(m), x_{j}(m), r_{j}(m), t_{j}(m), g_{j}, k_{j}, o_{j}, z_{j}, w_{j}$, are constants to determine. $M_{D_{i}}$ Is the mass of the molecule (drug), $\sigma_{D_{i}}$ is the el number of symmetry, $A B C$ : product of the moments of inertia of the ligand on the three main axes of rotation, $A B C$ : product of the moments of inertia of the ligand on the three main axes of rotation, $Q_{j}$ : net charge of the atom $j, S_{j}^{E}$ the total atomic electrophilic superdelocalizabilities of atom $j, S_{j}^{N}$ : is the total atomic nucleophilic superdelocalizabilities of atom $j, F_{j}(m)\left(F_{j}(m)\right)$ : is the electron population (Fukui index) of the occupied (vacant) MO m (m') localized on atom $j, S_{j}^{E}(m)$ is the atomic electrophilic superdelocalizability of MO m localized on atom $j$, $S_{j}^{N}(m)$ : is the atomic nucleophilic superdelocalizability of MO $\mathrm{m}^{\prime}$ localized on atom $j, \mu_{j}$ : is the local atomic electronic chemical potential of atom $j, \eta_{j}$ : Is the local atomic hardness of atom $j, \omega_{j}$ : Is the local atomic electrophilicity of atom $j \varsigma_{j}$ : Is the local atomic softness of atom $j, Q_{j}^{\max }$ : is the maximum amount of electronic charge that atom $j$ that atom $\mathrm{j}$ may accept from another site, $O_{k}$ : is the orientational parameters of the $\mathrm{k}_{\mathrm{th}}$ substituent $\mathrm{k}$.

Electronic populations are considered when assigning a localized molecular orbital to a specific atom. In this method, to assign a local OM to an atom, the latter must present electronic populations equal to or higher than 0.01 , according to Mulliken population analysis $[14,18]$. In this form, it is possible to find the local HOMO of atom $\mathrm{i}$ (that is, the highest occupied MO that has an electron population of 0.01 electron or more in atom i) and the local LUMO (usually denoted by HOMOi * and LUMOi $*$ respectively).

\section{SELECTION OF MOLECULES}

The selected molecules and their biological activities (Table [a]) were reported in a previous study [4]. The molecules under study have a common structure, where the substituents vary (Figure 1). The experimental results obtained in the reference study (biological activity) were obtained in vivo, and are expressed as a mean effective concentration (EC50). The EC50 indicates the concentrations in $\mu \mathrm{M}$ of the compound, which generate $50 \%$ of the allosteric modulation in the NTR1 receptor.

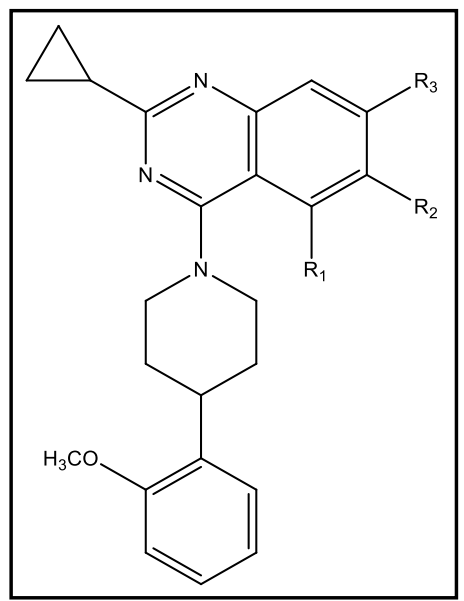

Figure 1. Common Structure. 
Table [a]. Selected molecules and their activities (EC50)

\begin{tabular}{|c|c|c|c|c|}
\hline Molecule & $\log E C 50$ & $\mathbf{R}_{1}$ & $\mathbf{R}_{2}$ & $\mathbf{R}_{3}$ \\
\hline 1 & 0.02 & $\mathrm{H}$ & $-\mathrm{OCH}_{3}$ & $\mathrm{OCH}_{3}$ \\
\hline 2 & -0.05 & $\mathrm{H}$ & $-\mathrm{N}\left(\mathrm{CH}_{3}\right)_{2}$ & $\mathrm{H}$ \\
\hline 3 & -0.25 & $\mathrm{H}$ & & $\mathrm{H}$ \\
\hline 4 & 0.83 & & $\mathrm{H}$ & $\mathrm{H}$ \\
\hline 5 & -0.14 & $\mathrm{H}$ & & $\mathrm{H}$ \\
\hline 6 & 1.00 & $\mathrm{H}$ & $\mathrm{H}$ & \\
\hline 7 & -0.01 & $\mathrm{H}$ & & $\mathrm{CH}_{3}$ \\
\hline 8 & 0.48 & $\mathrm{H}$ & & $\mathrm{F}$ \\
\hline 9 & 0.05 & $\mathrm{H}$ & & $\mathrm{H}$ \\
\hline 10 & -0.32 & $\mathrm{H}$ & & $\mathrm{H}$ \\
\hline 11 & -0.09 & $\mathrm{H}$ & & $\mathrm{H}$ \\
\hline 12 & 0.83 & $\mathrm{H}$ & & $\mathrm{H}$ \\
\hline 13 & 0.44 & $\mathrm{H}$ & & $\mathrm{H}$ \\
\hline 14 & -0.09 & $\mathrm{H}$ & & $\mathrm{H}$ \\
\hline 15 & -0.19 & $\mathrm{H}$ & & $\mathrm{H}$ \\
\hline 16 & 0.01 & $\mathrm{H}$ & & $\mathrm{H}$ \\
\hline 17 & -0.36 & $-\mathrm{H}$ & & $-\mathrm{H}$ \\
\hline
\end{tabular}




\section{CALCULATIONS}

The electronic structure of each molecule was obtained through the theory of functional density (DFT) at the level of theory B3LYP/6-31G (d, p), using Gaussian 09 software. Local reactivity indices were calculated using the DCENT-QSAR software [19].

For the group of molecules under study, the hypothesis of a typical skeleton was formulated (Figure 2). This hypothesis contains a set of atoms common to each of the molecules under study. Each atom of the common skeleton will be determined different indices of local reactivity, which will be related, through multiple regression analysis, with the logarithms of the biological activities. These results will allow obtaining the atoms that participate in the interaction with the receptor and the type of interaction involved. The variation of the values of a set of local reactivity indices, of one or more atoms belonging to the common skeleton, indicates the variation of the allosteric modulation in the NTR1 receptor $[14,19]$.

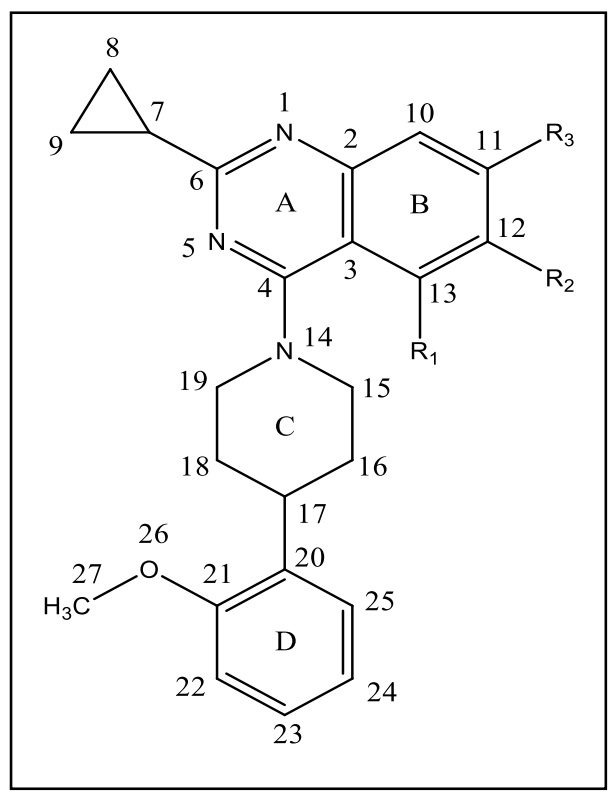

Figure 2. Common skeleton Numeration.

\section{RESULTS}

It is noteworthy to highlight that the reactivity indexes on the employed method are not normalized, given that they have a concrete physical meaning. This concrete physical meaning is particularly important to sustain the physical meaning of the equation and the comparison with studies in which different molecules are used in the interaction with the same receptor.
The method employed does not need external validation due to its formal mathematical structure [14].

\section{Results for the activity at NTR1 receptor}

The best statistically significant equation obtained for the set $\mathrm{I}$, is the following:

$L O G(E C 50)=16.80-42.49 S_{4}^{E}(H O M O-2)^{*}-48.17 F_{14}(H O M O-1)^{*}+52.66 S_{3}^{E} \quad$ (9) $+4.79 \mu_{21}+4.82 S_{11}^{E}(H O M O-2)^{*}-38.58 Q_{19}-6.67 F_{11}(L U M O+2)^{*}$

$+10.91 S_{1}^{N}(L U M O+1)^{*}$

with $\mathrm{n}=17, \mathrm{R}=0.98, \mathrm{R}^{2}=0.95$, adj. $\mathrm{R}^{2}=0.91, \mathrm{~F}(8,8)=21.21(\mathrm{p}<0.0001)$ and a standard error of estimate of 0.12 . No outliers were detected, and no residuals fall outside the $\pm 2 \sigma$ limits.

In equation $9 S_{4}^{E}(H O M O-2)^{*}$ it is the Fukui index of the second-highest occupied local molecular orbital in atom $4, F_{14}(H O M O-1)^{*}$ is the Fukui index of the second-highest occupied local molecular orbital in atom $14, S_{3}^{E}$ is the total electrophilic superdelocalizability in atom $3, \mu_{21}$ is the local electronic chemical potential in atom $21, S_{11}^{E}(H O M O-2)^{*}$ is the electrophilic superdelocalizability of the third-highest occupied local molecular in atom $11, Q_{19}$ is the net-charge located in atom $19, F_{11}(L U M O+2)^{*}$ is the Fukui index of the third lowest empty local molecular orbital in Atom $11, S_{1}^{N}(L U M O+1)^{*}$ is the nucleophilic superdelocalizability of the second-lowest empty local molecular orbital in atom 1.

Table [b]: Beta coefficients and t-test for significance of the coefficients in Eq. 9.

\begin{tabular}{|l|c|c|c|}
\hline & Beta & $\mathbf{t ~ ( 8 )}$ & p-level \\
\hline$S_{4}^{E}(H O M O-2)^{*}$ & -1.84 & -9.16 & $>0.00001$ \\
\hline$F_{14}(H O M O-1)^{*}$ & -1.89 & -7.65 & $>0.00006$ \\
\hline$S_{3}^{E}$ & 0.98 & 6.65 & $>0.0001$ \\
\hline$\mu_{21}$ & 0.67 & 5.03 & $>0.001$ \\
\hline$S_{11}^{E}(H O M O-2)^{*}$ & 0.49 & 4.33 & $>0.002$ \\
\hline$Q_{19}$ & -1.05 & -5.20 & $>0.0008$ \\
\hline$F_{11}(L U M O+2)^{*}$ & -0.48 & -3.41 & $>0.001$ \\
\hline$S_{1}^{N}(L U M O+1)^{*}$ & 0.50 & 3.05 & $>0.02$ \\
\hline
\end{tabular}

Squared correlation coefficients for the variables appearing in Eq.9.

Table [c]. Squared correlation coefficients for the variables appearing in Eq. 9.

\begin{tabular}{|c|c|c|c|c|c|c|c|}
\hline & $S_{4}^{E}(\mathrm{HOMO}-2)^{*}$ & $F_{14}(H O M O-1)^{*}$ & $S_{3}^{E}$ & $\mu_{21}$ & $S_{11}^{E}(H O M O-2)^{*}$ & $Q_{19}$ & $F_{11}(L U M O+2)^{*}$ \\
\hline$F_{14}(H O M O-1)^{*}$ & 0.21 & 1.00 & & & & & \\
\hline$S_{3}^{E}$ & 0.08 & 0.06 & 1.00 & & & & \\
\hline$\mu_{21}$ & 0.00 & 0.01 & 0.03 & 1.00 & & & \\
\hline$S_{11}^{E}(H O M O-2)^{*}$ & 0.08 & 0.28 & 0.05 & 0.22 & 1.00 & & \\
\hline$Q_{19}$ & 0.10 & 0.35 & 0.06 & 0.04 & 0.06 & 1.00 & \\
\hline$F_{11}(L U M O+2)^{*}$ & 0.29 & 0.01 & 0.05 & 0.12 & 0.11 & 0.23 & 1.00 \\
\hline$S_{1}^{N}(L U M O+1)^{*}$ & 0.36 & 0.00 & 0.04 & 0.14 & 0.00 & 0.07 & 0.24 \\
\hline
\end{tabular}




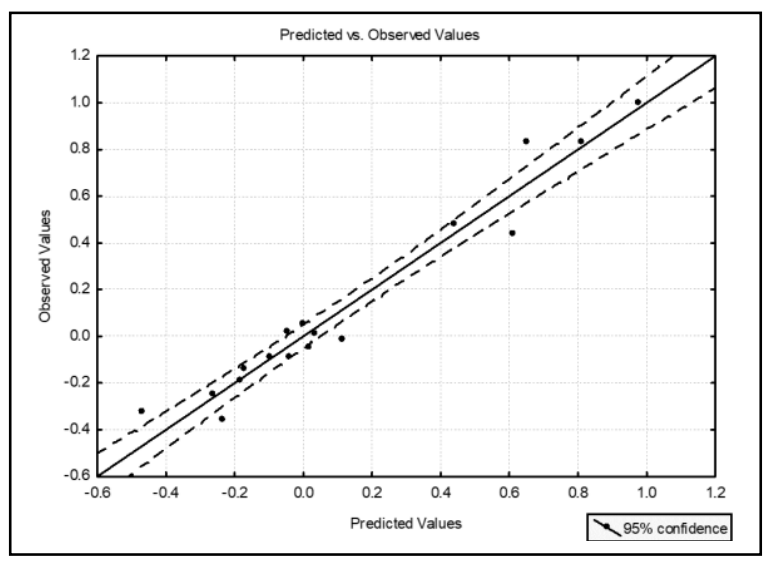

Figure 3. Plot of predicted vs. observed EC50 values (Eq. 9).

Table 3 shows the beta coefficients and the results of the t-test for the importance of coefficients in the equation. Table [c] shows low internal correlations between independent variables (the highest obtained value was 0.36). Figure 3 shows the graph of observed values versus calculated EC50 log values. Statistical data referring to the equation shows that equation 9 is statistically significant and represents the variation of 8 reactivity indices that belong to the common skeleton and accounts for approximately $91 \%$ of the variation in biological activity.

To propose the type of interaction for each atom of equation 9, the three highest local occupied molecular orbitals (HOMO)* and the three lowest local empty voids (LUMO)*. These molecular orbitals are shown in the following table.

Table [d]. Nomenclature: Molecule Set I (HOMO) / (HOMO-2) * (HOMO-1) * (HOMO) * - (LUMO) * (LUMO + 1) * (LUMO + 2) *.

\begin{tabular}{|c|c|c|c|c|}
\hline Mol. & Atom 1 & Atom 3 & Atom 4 & Atom 11 \\
\hline $1(112)$ & $109 \sigma 110 \sigma 112 \pi-113 \pi 114 \pi 117 \pi$ & $109 \sigma 110 \pi 112 \pi-113 \pi 114 \pi 117 \pi$ & $109 \sigma 110 \pi 112 \pi-113 \pi 114 \pi 117 \pi$ & $108 \pi 110 \pi 112 \pi-113 \pi 114 \pi 117 \pi$ \\
\hline $2(108)$ & $105 \sigma 106 \sigma 108 \pi-109 \pi 110 \pi 113 \pi$ & $105 \sigma 106 \pi 108 \pi-109 \pi 110 \pi 113 \pi$ & $105 \sigma 106 \pi 108 \pi-109 \pi 110 \pi 113 \pi$ & $104 \pi 106 \sigma 108 \pi-109 \pi 110 \pi 113 \pi$ \\
\hline $3(112)$ & $109 \sigma 110 \pi 112 \pi-113 \pi 114 \pi 117 \pi$ & $109 \sigma 110 \pi 112 \pi-113 \pi 114 \pi 117 \pi$ & $109 \sigma 110 \pi 112 \pi-113 \pi 114 \pi 117 \pi$ & $108 \sigma 110 \pi 112 \pi-113 \pi 114 \pi 117 \pi$ \\
\hline $4(116)$ & $113 \sigma 115 \pi 116 \pi-117 \pi 118 \sigma 121 \pi$ & $112 \pi 113 \sigma 115 \pi-117 \pi 118 \pi 121 \pi$ & $113 \sigma 115 \pi 116 \pi-117 \pi 118 \pi 121 \pi$ & $112 \pi 115 \pi 116 \pi-117 \pi 118 \pi 121 \pi$ \\
\hline $5(108)$ & $105 \sigma 106 \pi 108 \pi-109 \pi 110 \pi 113 \pi$ & $105 \sigma 106 \pi 108 \pi-109 \pi 110 \pi 113 \pi$ & $105 \sigma 106 \pi 108 \pi-109 \pi 110 \pi 113 \pi$ & $104 \pi 106 \pi 108 \pi-109 \pi 110 \pi 113 \pi$ \\
\hline $6(116)$ & $113 \sigma 115 \pi 116 \sigma-117 \pi 118 \pi 121 \pi$ & $113 \sigma 115 \pi 116 \pi-117 \pi 118 \pi 121 \pi$ & $112 \pi 113 \sigma 115 \pi-117 \pi 118 \pi 121 \pi$ & $109 \pi 110 \pi 116 \pi-117 \pi 118 \pi 121 \pi$ \\
\hline $7(120)$ & $118 \sigma 119 \pi 120 \pi-121 \pi 122 \pi 125 \pi$ & $117 \sigma 118 \sigma 120 \pi-121 \pi 122 \pi 125 \pi$ & $117 \sigma 118 \pi 120 \pi-121 \pi 122 \pi 125 \pi$ & $116 \pi 118 \pi 120 \pi-121 \pi 122 \pi 125 \pi$ \\
\hline $8(120)$ & $117 \sigma 118 \pi 120 \pi-121 \pi 122 \pi 125 \pi$ & $117 \sigma 118 \pi 120 \pi-121 \pi 122 \pi 125 \pi$ & $117 \sigma 118 \pi 120 \pi-121 \pi 125 \pi 126 \pi$ & $116 \pi 118 \pi 120 \pi-121 \pi 122 \pi 125 \pi$ \\
\hline $9(120)$ & $118 \pi 119 \pi 120 \pi-121 \pi 122 \pi 125 \pi$ & $117 \sigma 118 \pi 120 \pi-121 \pi 122 \pi 125 \pi$ & $117 \sigma 118 \pi 120 \pi-121 \pi 122 \pi 125 \pi$ & $116 \pi 118 \pi 120 \pi-121 \pi 122 \pi 125 \pi$ \\
\hline $10(116)$ & $113 \sigma 114 \pi 116 \pi-117 \pi 118 \pi 121 \pi$ & $113 \sigma 114 \pi 116 \pi-117 \pi 118 \pi 121 \pi$ & $113 \sigma 114 \pi 116 \pi-117 \pi 118 \pi 121 \pi$ & $112 \pi 114 \pi 116 \sigma-117 \pi 118 \pi 121 \pi$ \\
\hline $11(135)$ & $132 \sigma 134 \pi 135 \pi-136 \pi 137 \pi 140 \pi$ & $132 \pi 134 \pi 135 \pi-136 \pi 137 \pi 140 \pi$ & $132 \pi 134 \pi 135 \pi-136 \pi 137 \pi 140 \pi$ & $130 \pi 132 \pi 135 \pi-136 \pi 137 \pi 140 \pi$ \\
\hline $12(123)$ & $120 \sigma 121 \sigma 123 \pi-124 \pi 125 \pi 128 \pi$ & $120 \sigma 121 \pi 123 \pi-124 \pi 125 \pi 126 \pi$ & $120 \sigma 121 \pi 123 \pi-124 \pi 125 \pi 128 \pi$ & $119 \pi 121 \pi 123 \pi-124 \pi 125 \pi 126 \pi$ \\
\hline $13(127)$ & $125 \sigma 126 \pi 127 \pi-128 \pi 129 \pi 132 \pi$ & $124 \sigma 125 \pi 127 \pi-128 \pi 129 \pi 130 \pi$ & $124 \sigma 125 \pi 127 \pi-128 \pi 129 \pi 132 \pi$ & $123 \pi 125 \pi 127 \pi-128 \pi 129 \pi 130 \pi$ \\
\hline $14(123)$ & $121 \pi 122 \pi 123 \pi-124 \pi 125 \pi 128 \pi$ & $120 \pi 121 \pi 123 \pi-124 \pi 125 \pi 128 \pi$ & $120 \pi 121 \pi 123 \pi-124 \pi 125 \pi 128 \pi$ & $119 \pi 121 \pi 123 \pi-124 \pi 125 \pi 128 \pi$ \\
\hline $15(123)$ & $120 \sigma 121 \pi 123 \pi-124 \pi 125 \pi 128 \pi$ & $120 \sigma 121 \pi 123 \pi-124 \pi 125 \pi 126 \pi$ & $120 \sigma 121 \pi 123 \pi-124 \pi 125 \pi 128 \pi$ & $119 \pi 121 \pi 123 \pi-124 \pi 125 \pi 126 \pi$ \\
\hline $16(119)$ & $116 \sigma 117 \sigma 119 \pi-120 \pi 121 \pi 124 \pi$ & $116 \sigma 117 \pi 119 \pi-120 \pi 121 \pi 124 \pi$ & $116 \sigma 117 \pi 119 \pi-120 \pi 121 \pi 124 \pi$ & $115 \pi 117 \pi 119 \pi-120 \pi 121 \pi 124 \pi$ \\
\hline $17(119)$ & $116 \sigma 117 \pi 119 \pi-120 \pi 121 \pi 124 \pi$ & $116 \sigma 117 \pi 119 \pi-120 \pi 121 \pi 124 \pi$ & $116 \sigma 117 \pi 119 \pi-120 \pi 121 \pi 124 \pi$ & $115 \pi 117 \pi 119 \pi-120 \pi 121 \pi 124 \pi$ \\
\hline
\end{tabular}

\begin{tabular}{|c|c|c|c|}
\hline Mol. & Atom 14 & Atom 19 & Atom 21 \\
\hline $1(112)$ & $109 \pi 110 \pi 112 \sigma-113 \pi 117 \pi 119 \pi$ & $109 \sigma 110 \sigma 112 \sigma-113 \sigma 117 \sigma 119 \sigma$ & $102 \sigma 107 \pi 111 \pi-115 \pi 116 \pi 118 \pi$ \\
\hline $2(108)$ & $105 \sigma 106 \sigma 108 \sigma-109 \sigma 113 \sigma 115 \pi$ & $103 \sigma 104 \sigma 106 \sigma-109 \sigma 115 \sigma 121$ & $98 \sigma 104 \pi 107 \pi-111 \pi 112 \pi 114--$ \\
\hline $3(112)$ & $110 \sigma 111 \pi 112 \sigma-113 \pi 117 \pi 120 \pi$ & $102 \sigma 108 \sigma 110 \sigma-113 \sigma 120 \sigma 124 \sigma$ & $108 \pi 110 \sigma 111 \pi-115 \pi 116 \pi 119 \pi$ \\
\hline $4(116)$ & $113 \pi 115 \pi 116 \pi-117 \sigma 121 \sigma 122 \sigma$ & $112 \sigma 115 \sigma 116 \sigma-117 \sigma 121 \sigma 123 \sigma$ & $106 \sigma 111 \pi 114 \pi-119 \pi 120 \pi 124 \pi$ \\
\hline $5(108)$ & $106 \pi 107 \pi 108 \sigma-109 \pi 113 \pi 117 \pi$ & $104 \sigma 106 \sigma 107 \sigma-109 \sigma 111 \sigma 116 \sigma$ & $104 \sigma 106 \sigma 107 \pi-111 \pi 112 \pi 115 \pi$ \\
\hline $6(116)$ & $112 \pi 113 \sigma 115 \pi-117 \pi 121 \pi 125 \pi$ & $106 \pi 112 \sigma 115 \sigma-117 \sigma 119 \sigma 121 \sigma$ & $106 \sigma 111 \pi 114 \pi-119 \pi 120 \pi 123 \pi$ \\
\hline $7(120)$ & $118 \sigma 119 \pi 120 \sigma-121 \pi 125 \pi 127 \pi$ & $116 \sigma 118 \sigma 119 \sigma-121 \sigma 123 \sigma 125 \sigma$ & $115 \pi 118 \pi 119 \pi-123 \pi 124 \pi 127 \pi$ \\
\hline $10(116)$ & $114 \sigma 115 \pi 116 \sigma-117 \pi 121 \pi 125 \pi$ & $112 \sigma 114 \sigma 115 \sigma-117 \sigma 119 \sigma 121 \sigma$ & $112 \pi 114 \pi 115 \pi-119 \pi 120 \pi 124 \pi$ \\
\hline $10(116)$ & $114 \sigma 115 \pi 116 \sigma-117 \pi 121 \pi 125 \pi$ & $112 \sigma 114 \sigma 115 \sigma-117 \sigma 119 \sigma 121 \sigma$ & $112 \pi 114 \pi 115 \pi-119 \pi 120 \pi 124 \pi$ \\
\hline $10(116)$ & $114 \sigma 115 \pi 116 \sigma-117 \pi 121 \pi 125 \pi$ & $112 \sigma 114 \sigma 115 \sigma-117 \sigma 119 \sigma 121 \sigma$ & $112 \pi 114 \pi 115 \pi-119 \pi 120 \pi 124 \pi$ \\
\hline $11(135)$ & $133 \pi 134 \pi 135 \sigma-136 \pi 140 \pi 146 \pi$ & $130 \sigma 132 \sigma 133 \pi-136 \sigma 138 \sigma 140 \sigma$ & $130 \pi 132 \pi 133 \pi-138 \pi 139 \pi 142 \pi$ \\
\hline $12(123)$ & $121 \sigma 122 \pi 123 \sigma-124 \pi 128 \pi 133 \pi$ & $112 \sigma 119 \sigma 121 \sigma-124 \sigma 132 \sigma 135 \sigma$ & $119 \pi 121 \sigma 122 \pi-126 \pi 127 \pi 131 \pi$ \\
\hline $13(127)$ & $125 \sigma 126 \pi 127 \sigma-128 \sigma 132 \pi 137 \pi$ & $123 \sigma 125 \sigma 126 \sigma-128 \sigma 136 \sigma 141 \sigma$ & $123 \pi 125 \pi 126 \pi-130 \pi 131 \pi 134 \pi$ \\
\hline $14(123)$ & $121 \sigma 122 \pi 123 \sigma-124 \pi 128 \pi 133 \pi$ & $119 \sigma 121 \sigma 122 \sigma-124 \sigma 126 \sigma 128 \sigma$ & $119 \pi 121 \pi 122 \pi-126 \pi 127 \pi 131 \pi$ \\
\hline $15(123)$ & $121 \sigma 122 \pi 123 \sigma-124 \pi 128 \sigma 133 \pi$ & $112 \sigma 119 \sigma 121 \sigma-124 \sigma 128 \sigma 132$ & $119 \pi 121 \pi 122 \pi-126 \pi 127 \pi 131 \pi$ \\
\hline $16(119)$ & $116 \sigma 117 \sigma 119 \sigma-120 \pi 124 \pi 127 \pi$ & $108 \sigma 115 \sigma 117 \sigma-120 \sigma 124 \sigma 127 \sigma$ & $108 \sigma 114 \pi 118 \pi-122 \pi 123 \pi 128 \pi$ \\
\hline $17(119)$ & $117 \pi 118 \pi 119 \sigma-120 \pi 124 \pi 127 \pi$ & $115 \sigma 117 \sigma 118 \sigma-120 \sigma 122 \sigma 124 \sigma$ & $115 \pi 117 \pi 118 \pi-122 \pi 123 \pi 126 \pi$ \\
\hline
\end{tabular}




\section{DISCUSSION}

The beta values represented in Table 2 show that the order of importance of the variables follows the subsequent order:

$$
\begin{aligned}
& F_{14}(\text { HOMO }-1)^{*}>S_{4}^{E}(\text { HOMO }-2)^{*}>Q_{19}>S_{3}^{E}>\mu_{21}>S_{1}^{N}(L U M O+1)^{*}> \\
& S_{11}^{E}(H O M O-2)^{*} \approx F_{11}(L U M O+2)^{*}
\end{aligned}
$$

The process is governed by weak interactions that depend on the electronic populations and the energy of the OMs for $\mathrm{N} 1, \mathrm{C} 3, \mathrm{C} 4, \mathrm{C} 11, \mathrm{C} 14, \mathrm{C} 21$, while for C19 (Figure 2), it depends on the net charge on this atom.

The variable $S_{1}^{N}(L U M O+1)^{*}$ will not be discussed due to the high value of $\mathrm{p}$ (Table $[b]$ ).

The discussion of the results will be conveyed knowing that: Fukui indices are always positive, total and partial electrophilic superdelocalizabilities are negative, the charge of atom 19 is positive, and the electronic chemical potential is always negative.

Atom 14 is a nitrogen of ring $\mathrm{C}$ (Figure 2). High values of $F_{14}(H O M O-1)^{*}$ are associated with high biological activity. According to Table [d], its OMs are of $\sigma o \pi$ nature. Most of the local OM molecules coincides with molecular HOMO, which indicates a high reactivity of the local OM. It is suggested that atom 14 is interacting with a deficient center in electrons through its first two busiest local molecular orbitals $[20,21]$.

Atom 4 is a carbon of aromatic ring A (Figure 2). Lower negative values of $S_{4}^{E}(H O M O-2)^{*}$ can be associated with high biological activity. This carbon is attached to two nitrogen atoms, N5 (ring A) and N14 (ring C). The OM $(\mathrm{HOMO})^{*}$, (HOMO-1) $)^{*}$, (HOMO-2) $)^{*}$, are of $\pi$ nature, where local HOMO coincides with the molecular HOMO (Table [d]). The high electronegativity of nitrogen would decrease the electron density in the carbon atom 4. It is suggested that atom 4 interacts with a rich center in electrons [22, 23].

Atom 3 is a carbon that links rings A and B (Figure 2). High negative values of $S_{3}^{E}$ are related to a high activity of receptor NTR1. Table [d] shows local OMs of this carbon are of $\pi$ nature. It is suggested that this atom interacts with an electron-deficient $\pi$ center [22].

Atom 21 is an atom of carbon attached to O26 (metoxi group) (Figure 2). A high affinity of the receptor is associated with negative values for $\mu_{21}$. This index is the middle point between energies (HOMO) * and (LUMO) *. Table [d] shows that all local MO have a $\pi$ nature, (LUMO) * is energetically far from molecular LUMO and (HOMO) * is very close to molecular HOMO. Therefore, to obtain more negative values for $\mu$, a substituent can be attached to the atom 21 such that LUMO o LUMO + 1 of molecule localized in this atom, making empty MO more reactive, improving its capacity to take electrons. It is suggested that atom 21 is probably interacting with a site (atom or residue) with a busy OM $\sigma$ (sometimes called the apolar region) $[14,23]$.

Atom 19 is a carbon of the saturated ring C (Figure 2). Negative values of $Q_{19}$ associated to a high biological activity. Its local OMs are of $\sigma$ nature (Table [d]). This carbon is attached to a nitrogen atom, which would mean that the high electronegativity of the latter would decrease the electronic density of carbon 19. It is suggested that this atom interacts by electrostatic bonding with a negatively charged center [24-26].

A particular situation results in the analysis of the results for atom 11. This atom, is a carbon of the aromatic ring B (Figure 2). According to Table [d], its local OMs are of $\pi$ nature. The fact that they relate to the high activity of NTR1 high values of $S_{11}^{E}(H O M O-2)^{*}$ and $F_{11}(L U M O+2)^{*}$ implies that this carbon interacts with a deficient center of electrons in the first case and a rich electrons center in the second case, at the same time [27, 28]. It is suggested that for $S_{11}^{E}(H O M O-2)^{*}$ the interaction occurs with empty OM from a receptor site; meanwhile, for $F_{11}(L U M O+2)^{*}$ the interaction occurs with a busy OM. $(\mathrm{HOMO}-2)^{*}$ and $(\mathrm{LUMO}+2)^{*}$ are of $\pi$ nature in atom 11 , where local HOMO and LUMO coincide with molecular HOMO and LUMO. This suggests that, in the case of $F_{11}(L U M O+2)^{*}$ a busy OM overlap of the receptor would occur which increases the electronic density over carbon, while in $S_{11}^{E}(H O M O-2)^{*}$ could generate an interaction by giving electronic density to a receptor site by interaction $\pi-\pi$ or $\sigma-\pi$, which will allow stabilizing the electronic density on itself.
Both facts are compatibles. Another possible interpretation of this situation is a significant difference in the beta values or a high correlation between both variables. Nevertheless, this situation does not occur, as can be seen in Table 3 and in Table $4[29,30]$.

These results are represented in Figure 4.

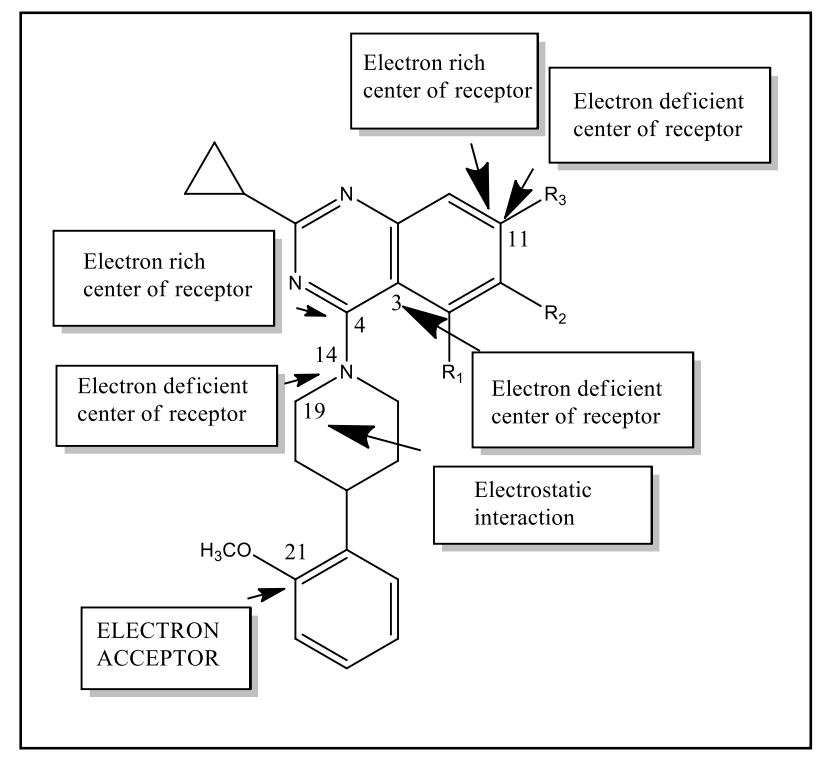

Figure 4. 2D pharmacophore for receptor binding NTR1.

\section{CONCLUSIONS}

Through the use of LMRA, a statistically significant equation was obtained for the variation of the biological activity generated by quinazoline derivatives, concerning the variation of a set of local atomic reactivity indices. From the results, a 2D pharmacophore was proposed, in which it is shown that the biological process is governed mainly by weak interactions at the orbital-orbital level and by loads. These results may facilitate the synthesis of quinazolinederived drugs, with high allosteric modulation (antagonist) in the NTR1 receptor.

\section{ACKNOWLEDGEMENTS}

This work was financially supported by project UTA Mayor $\mathrm{N}^{0} 4753-19$. The authors would like to thank Dr. María Camarada, Director of the Center for Applied Nanotechnology, Faculty of Sciences Universidad Mayor for providing the means to carry out this study.

\section{REFERENCES}

1. Kato HE, Zhang Y, Hu H, Suomivuori C-M, Kadji FMN, Aoki J, et al. Conformational transitions of a neurotensin receptor $1-\mathrm{G}$ i1 complex. Nature 2019:1.

2. Hwang JR, Baek MW, Sim J, Choi H-S, Han JM, Kim YL, et al Intermolecular cross-talk between NTR1 and NTR2 neurotensin receptor promotes intracellular sequestration and functional inhibition of NTR1 receptors. Biochem Bioph Res Co 2010;391:1007-13.

3. Choi S-Y, Chae H-D, Park T-J, Ha H, Kim K-T. Characterization of high affinity neurotensin receptor NTR1 in HL-60 cells and its down regulation during granulocytic differentiation. Brit J Pharmacol 1999;126:1050.

4. Pinkerton AB, Peddibhotla S, Yamamoto F, Slosky L, Bai Y, Maloney PR, et al. Discovery of b-Arrestin Biased, Orally Bioavailable and CNS Penetrant Neurotensin Receptor 1 (NTR1) Allosteric Modulators. J Med Chem 2019.

5. Onaga T, Shimoda T, Ohishi T, Yasui Y, Hayashi H. Role of neurotensin in the regulation of gastric motility in healthy conscious sheep. Small Ruminant Res 2019;172:31-41.

6. Labbé-Jullié C, Barroso S, Nicolas-Etève D, Reversat J-L, Botto J-M, Mazella J, et al. Mutagenesis and modeling of the neurotensin receptor NTR1 identification of residues that are critical for binding SR 48692, a nonpeptide neurotensin antagonist. J Med Chem 1998;273:16351-7.

7. Pelaprat D. Interactions between neurotensin receptors and $G$ proteins. Peptides 2006; 27:2476-87. 
8. Ye Y, Long X, Zhang L, Chen J, Liu P, Li H, et al. NTS/NTR1 co-expression enhances epithelial-to-mesenchymal transition and promotes tumor metastasis by activating the $\mathrm{Wnt} / \beta$-catenin signaling pathway in hepatocellular carcinoma. Oncotarget 2016;7:70303.

9. Gahbauer S, Pluhackova K, Böckmann RA. Closely related, yet unique: Distinct homo-and heterodimerization patterns of $\mathrm{G}$ protein coupled chemokine receptors and their fine-tuning by cholesterol. Plos Comput Biol 2018;14:e1006062.

10. Griebel G, Holsboer F. Neuropeptide receptor ligands as drugs for psychiatric diseases: the end of the beginning? Nat Rev Drug Discov 2012;11:462.

11. Gómez-Jeria JS. 45 Years of the KPG Method: A Tribute to Federico Peradejordi. Journal of Computational Methods in Molecular Design 2017;7:17-37.

12. Muñoz-Gacitúa D, Gómez-Jeria JS. Quantum-chemical study of the relationships between electronic structure and anti-influenza activity. 2 . The inhibition by $1 \mathrm{H}-1,2,3$-triazole-4-carboxamide derivatives of the cytopathic effects produced by the influenza A/WSN/33 (H1N1) and A/HK/8/68 (H3N2) strains in MDCK cells. Journal of Computational Methods in Molecular Design 2014;4:48-63.

13. Gómez-Jeria JS. A quantum chemical analysis of the relationships between electronic structure, PAK1 inhibition and MEK phosphorylation in a series of 2-arylamino-4-aryl-pyrimidines. SOP Trans Phys Chem 2014;1:10-28.

14. Kpotin GA, Bédé AL, Houngue-Kpota A, Anatovi W, Kuevi UA, Atohoun GS, et al. Relationship between electronic structures and antiplasmodial activities of xanthone derivatives: a 2D-QSAR approach. Stuc Chem 2019:110.

15. Gómez-Jeria JS. A new set of local reactivity indices within the HartreeFock-Roothaan and density functional theory frameworks. Can Chem Trans 2013;1:25-55.

16. Gómez-Jeria J. Calculation of the nucleophilic superdelocalizability by the CNDO/2 method. J Pharm Sci 1982;71:1423.

17. Gómez-Jeria JS. Modeling the drug-receptor interaction in quantum pharmacology. Molecules in Physics, Chemistry, and Biology: Springer; 1989; 215-31.

18. Gautier, Kankinou \& Kpotin, Gaston \& Mensah, Jean-Baptiste \& GómezJeria, Juan-Sebastián.. Quantum-Chemical Study of the Relationships between Electronic Structure and the Affinity of Benzisothiazolylpiperazine Derivatives for the Dopamine Hd2l and Hd3 Receptors. Pharm. Chem. J. 2019; 6: 73-90.

19. Gómez-Jeria JS. An empirical way to correct some drawbacks of mulliken population analysis. J. Chil. Chem. Soc 2009;54:482-5.

20. Kpotin GA, Gómez-Jeria JS. A Quantum-chemical Study of the Relationships Between Electronic Structure and Anti-proliferative Activity of Quinoxaline Derivatives on the HeLa Cell Line. Chemistry 2017;5:59-68.
21. Soro D, Ekou L, Ouattara B, Kone MG-R, Ekou T, Ziao N. DFT Study, Linear and Nonlinear Multiple Regression in the Prediction of HDAC7 Inhibitory Activities on a Series of Hydroxamic Acids. Comput Mol Biosci 2019;9:63.

22. Valdebenito-Gamboa J, Gómez-Jeria JS. A theoretical analysis of the relationships between electronic structure and HIV-1 integrase inhibition and antiviral activity of a series of naphthyridinone derivatives. Der Pharma Chem 2015;7:543-55.

23. Gómez-Jeria JS, Cornejo-Martínez R. A DFT study of the inhibition of human phosphodiesterases PDE3A and PDE3B by a group of 2-(4- $(1 \mathrm{H}-$ tetrazol-5-yl)-1H-pyrazol-1-yl)-4-(4-phenyl) thiazole derivatives. Der Pharma Chem 2016;8:329-37.

24. Soloaga Ardiles C, Cárcamo Vega J. Theoretical analysis of the relationship between the electronic structure and its inhibitory action in the $\mathrm{p} 2 \mathrm{x} 7 \mathrm{r}$ receptor of a series of 2-hydroxy-1, 4-naphthoquinones derivatives. J. Chil. Chem. Soc 2018;63:4205-10.

25. Gómez-Jeria JS, Valdebenito-Gamboa J. A quantum-chemical analysis of the antiproliferative activity of N-3-benzimidazolephenylbisamide derivatives against MGC803, HT29, MKN45 and SW620 cancer cell lines. Der Pharma Chem 2015;7:103-21.

26. Kpotin G, Atohoun SY, Kuevi A, Kpota-Hounguè A, Mensah J-B, Jeria JG. A Quantum-Chemical study of the Relationships between Electronic Structure and Trypanocidal Activity against Trypanosoma Brucei Brucei of a series of Thiosemicarbazone derivatives. Pharm Lett 2016;8:215-22.

27. Gómez Jeria J, Kpotin GA. Some remarks on the interpretation of the local atomic reactivity indices within the Klopman Peradejordi Gomez (KPG) method. I. Theoretical analysis. 2018.

28. Anatovi W, Kpotin GA, Kuevi UA, Houngue-Kpota A, Atohoun GS, Mensah $\mathrm{J}-\mathrm{B}$, et al. A DFT study or the relationship between the electronic structure and the antiplasmodial activity of a series of 4-anilino-2trichloromethylquinazolines derivatives. World Sci News 2017;88:138-51.

29. Gómez-Jeria J, Valdebenito-Gamboa J. A DFT Study of the Relationships between the Electronic Structures of a series of 2, 4, 5-Trisubstituted Pyrimidines and their Inhibition of four Cyclin-dependent Kinases and their Anti-Proliferative Action against HCT-116 and MCF-7 Cell Lines. Der Pharma Chem 2014;6:383-406.

30. Gómez-Jeria JS, Abarca-Martínez S. A theoretical approach to the cytotoxicity of a series of $\beta$-carbolinedithiocarbamate derivatives against prostatic cancer (DU-145), breast cancer (MCF-7), human lung adenocarcinoma (A549) and cervical cancer (HeLa) cell lines. Der Pharma Chem 2016;8:507-26. 